\title{
Lokale und systemische Mykosen
}

W ahrscheinlich täglich haben wir kontakt mit Pilzen, die als Saprophyten in der Umwelt existieren (z.B. Schimmelpilze) oder als Kommensalen unsere Haut und Schleimhaut besiedeln (Candida-Hefen). Trotz oder gerade wegen der Allgegenwärtigkeit der Pilze ist kaum ein anderes Thema der Infektiologie in der Bevölkerung - aber auch in der Fachwelt - so umstritten wie Infektionen durch Pilze. Die Dimension reicht von der als harmlos bewerteten Besiedlung der Mukosa bis hin zur Mykophobie des vermeintlich „verpilzten Patienten“. Immer wieder finden sich Themen wie die Pilzexposition durch Biotonnen bzw. Kompostierungsanlagen oder die Frage, welche Lüftungsstrategie einem Schimmelpilzbefall von Wohnräumen am besten vorbeugen kann. Der Verkauf publikumswirksamer Buchtitel trägt sicherlich nicht unwesentlich zur Verunsicherung von Laien und selbsternannten Experten bei. Schuldzuweisungen sind jedoch unangebracht, ist doch unser Wissen um pathogenetische Zusammenhänge von Mykosen bisher sehr limitiert.

Außer den Dermatophyten und den Erregern einer außereuropäischen Systemmykose (z.B. Histoplasma, Coccidioides) werden Pilze als fakultativ pathogen eingestuft. Dementsprechend ist auch das Miteinander von Pilz und Mensch vielseitig: Die Palette reicht von der Besiedlung von Haut oder Schleimhäuten gesunder Individuen über oberflächliche Schleimhautmykosen bis hin zu Systemmykosen. Gerade systemische Infektionen gewinnen vor dem Hintergrund zunehmender Zahlen von Patienten mit Immunschwäche an Bedeutung. Gefährdet sind dabei vor allem Patienten mit hämatologisch-onkologischen Grunderkrankungen und Neutropenie sowie solche, die sich einer immunsuppressiven Therapie nach Organtransplantation unterziehen müssen. Auf Intensivstationen ist Candida albicans mittlerweile der vierthäufigste Infektionserreger: Alleine in Deutschland führt er zu mehreren Tausend Todesfällen pro Jahr. Auch systemische Aspergillosen sind nach wie vor mit einer sehr hohen Letalität belastet (70-90\%).

Die Letalität der systemischen Mykosen ist aus dreierlei Gründen sehr hoch: Erstens sind die Patienten in der Regel immunsupprimiert, zweitens ist die mikrobiologische Diagnostik bisher unzureichend und drittens sind die Therapiemöglichkeiten begrenzt. Daher wird bei immunsupprimierten Patienten inzwischen meist eine empirische antimykotische Therapie eingeleitet. Bis zu 60\% der Patienten erhalten nach intensiver immunsuppressiver Therapie auf Verdacht hin teure und oft sehr toxische antimykotische Medikamente. Es verwundert daher nicht, dass die Kosten für Antimykotika inzwischen in vielen hämatologischen Abteilungen den Hauptposten in den Arzneimittelbudgets darstellen.

So hat sich die klinische Mykologie in der Zwischenzeit zu einem regelrechten „Markt der Therapie“ entwickelt: Antimykotika neuer Wirkstoffklassen stehen zur Verfügung und das Interesse der Pharmafirmen an neuen potenziellen Zielstrukturen der Pilze ist offensichtlich. Doch vor der Therapie kommt bekanntlich die Diagnose - trotz Einzug molekularbiologischer Techniken nicht selten eher noch als Fehldiagnose zu bewerten.

Im Widerspruch zur klinischen und sozioökonomischen Bedeutung lokaler und systemischer Mykosen steht die Tatsache, dass die mykologische Forschung in Deutschland bisher nur begrenzt gefördert wird. Vor allem die Identifizierung jener Mechanismen, die den Übergang von der Kolonisation hin zur Infektion bewirken, fordern interdisziplinär denkende Wissenschaftler und Kliniker. Die Genome wichtiger Pilzarten sind bereits sequenziert oder werden es demnächst sein. Dies ist die Voraussetzung, um die Infektionsmechanismen von humanpathogenen Pilzen auf breiter Basis analysieren zu können. Außerdem wurden in den letzten Jahren Methoden entwickelt, die Pilze einer genetischen Manipulation zugänglich machen, was die Identifikation von für die Erkrankung essenziellen Faktoren auf Seiten des Pilzes möglich macht. Dadurch lassen sich in Zukunft auch Zielstrukturen erkennen, mit deren Hilfe hoffentlich die Diagnostik, Therapie und Prävention von Mykosen beim Menschen verbessert werden kann.

Die Mykologie steht also an der Schwelle zu neuen Herausforderungen. Dass auch die Politik die Bedeutung mykologischer Erkrankungen erkannt hat, ist nicht zuletzt daran zu erkennen, dass trotz knapper Kassen erst kürzlich ein „Nationales Referenzzentrum für Systemische Mykosen“ durch das Bundesministerium für Gesundheit berufen wurde. Es bleibt zu hoffen, dass Klinik, medizinische Mikrobiologie und Grundlagenforschung dieses Angebot annehmen und zueinander finden, um gemeinsam den Herausforderungen der Mykologie begegnen zu können.
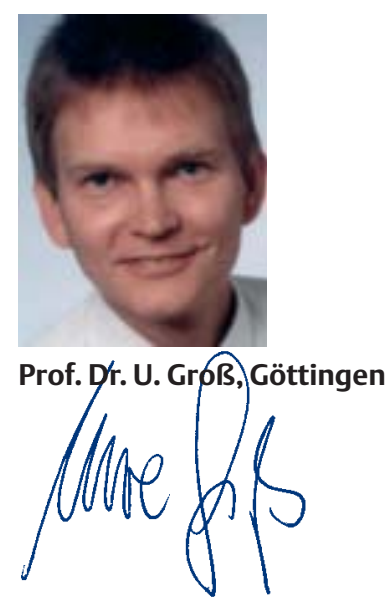